\title{
Long term safety, efficacy, and patient acceptability of hyaluronic acid injection in patients with painful osteoarthritis of the knee
}

This article was published in the following Dove Press journal:

Patient Preference and Adherence

II December 2012

Number of times this article has been viewed

\section{Benjamin A McArthur Christopher J Dy \\ Peter D Fabricant Alejandro Gonzalez Della Valle}

Department of Orthopedic Surgery, Hospital for Special Surgery, New York, NY, USA
Correspondence: Christopher J Dy 535 E 70th Street, New York, NY, USA $\mathrm{Tel}+|2| 2606$ |466

Fax +I 2I2606 I477

Email dyc@hss.edu

\begin{abstract}
The increasing prevalence of painful knee osteoarthritis has created an additional demand for pharmacologic management to prevent or delay surgical management. Viscosupplementation, via intraarticular injection of hyaluronic acid (HA), aims to restore the favorable milieu present in the nonarthritic joint. The safety profile of intraarticular HA injections for painful knee osteoarthritis is well established, with the most common adverse effect being a self-limited reaction at the injection site. Although acceptance of the early literature has been limited by publication bias and poor study quality, more recent and rigorous meta-analysis suggests that intraarticular HA injection is superior to placebo injection for pain relief and matches, if not surpasses, the effect size of other nonoperative treatments, such as nonsteroidal anti-inflammatory medication. Intraarticular HA injection is effective in providing temporary pain relief in patients with painful knee osteoarthritis. Future investigations should focus on optimizing the composition and administration of HA agents to provide prolonged relief of painful osteoarthritis in the knee and other joints.
\end{abstract}

Keywords: intraarticular injection, hyaluronate, viscosupplementation, osteoarthritis, knee

\section{Introduction}

Osteoarthritis (OA) is a chronic, progressive disease which produces significant pain and disability. A survey of causes for lost productivity in the United States workforce identified arthritis as a major cause of lost work time, second only to back pain, and the primary cause of reduced performance at work. ${ }^{1}$ Advanced age and obesity are major risk factors for the development of $\mathrm{OA}$, and with current population trends demonstrating significant increases in both, the prevalence of OA is expected to increase substantially in the coming years. Recent projections suggest that, by 2030, 67 million Americans, approximately $25 \%$ of the adult population, will be affected by OA. ${ }^{2}$ While OA remains a disease affecting the elderly, with over half of cases occurring in those more than 65 years old, it is estimated that working-age adults (ages 45-64 years) will represent one-third of cases in the coming years. ${ }^{2}$

While prosthetic total-knee replacement can effectively treat arthritis-mediated pain, nonoperative treatment modalities can serve to delay the need for surgical intervention or manage patient symptoms among those who are not ideal surgical candidates. Nonoperative measures include lifestyle modifications, physical therapy, nonsteroidal anti-inflammatory drugs (NSAIDs), other local or systemic analgesic agents, intraarticular corticosteroid injections, and viscosupplementation with hyaluronic acid (HA). Intraarticular injection of HA is an increasingly popular therapy for knee OA and is supported by a growing body of literature. ${ }^{3-5}$ This article reviews the current literature 
pertaining to viscosupplementation, with specific focus on the biology, safety, efficacy, and patient-acceptability profile.

\section{Biology}

Viscosupplementation involves intraarticular injection of HA, the viscoelastic mucopolysaccharide component of synovial fluid, after aspiration of any existing joint effusion. HA, also referred to as hyaluronate or hyaluronan, is a high-molecular weight glycosaminoglycan that consists of a repeating sequence of disaccharide units composed of $\mathrm{N}$-acetyl glucosamine and glucuronic acid. ${ }^{5,6}$ It is produced by type B synoviocytes and synovial fibroblasts and is secreted into the joint, where it serves as a lubricant, shock absorber, extracellular matrix scaffold, and chondroprotective milieu, to facilitate chondrocyte nutrition and to curb inflammation. ${ }^{4-7}$

Compared with the healthy adult knee, the osteoarthritic knee is characterized by reductions in both the concentration and molecular weight of HA as a result of synovial fluid dilution with effusion, abnormal synoviocyte production, and molecular fragmentation. ${ }^{5,6,8}$ Increased levels of proinflammatory cytokines, free radicals, and proteinases alter HA synthesis and reduce the viscoelastic properties of synovial fluid in the arthritic knee. ${ }^{4}$ Furthermore, enzymatic cleavage of ultrahigh molecular weight HA molecules into lower-weight HA has been noted to induce proinflammatory pathways, which may accelerate the progression of disease. ${ }^{4,5,9,10}$ Increased levels of excitatory amino acid neurotransmitters glutamate and aspartate have been implicated in the pathogenesis of early $\mathrm{OA}$ in a rat model..$^{11}$ Interestingly, Jean et $\mathrm{a}{ }^{12}$ demonstrated a decrease in glutamate and aspartate levels in their anterior cruciate ligament (ACL)-transected rat model after intraarticular injection of hyaluronic acid. More recently, Tsai et al ${ }^{13}$ noted slowing of osteoarthritic progression after intraarticular HA in this animal model, with better cartilage and synovium condition seen in combination with decreased glutamate levels, compared with saline-injected controls. Thus, viscosupplementation aims to restore the favorable milieu created by high-molecular weight HA. Though the exact mechanism of action is not known, HA injection is thought to exert its effect both through direct mechanical protection of the articular surface and through a number of metabolic effects, including induction of endogenous HA production, reduction in synovial inflammation, and possible promotion of chondrocyte proliferation and differentiation. ${ }^{14,15}$

Pharmaceutical production of HA has been achieved primarily by two methods: extraction and purification from rooster combs; and purified biologic fermentation from bacterial culture..$^{5,7,8}$ The former can be modified through cross-linking, to increase the molecular weight of the product. Differences in production techniques result in substantial variation in molecular weight of the products, and the clinical significance of this remains a source of continued debate. ${ }^{3}$ While some have reported no correlation between molecular weight and efficacy, ${ }^{16}$ others have attributed greater effects to HA preparations with higher molecular weight, based on both clinical ${ }^{17}$ and in vitro data. ${ }^{18}$ The differences in molecular weight of HA preparations have limited the ability of meta-analyses to provide conclusive data regarding the effectiveness of intraarticular HA because of the high amount of study heterogeneity. ${ }^{19}$

\section{Safety}

The safety profile for intraarticular HA injections has been well defined in a number of large level 1 studies, with a recent Cochrane review demonstrating a favorable safety profile (aside from local adverse events such as transient pain and swelling at the injection site). ${ }^{3}$ It is important to note that many, if not nearly all, of the trials evaluating intraarticular HA are funded by the pharmaceutical industry, which may introduce bias into the analysis and interpretation of the results.

While no major systemic safety issues have been detected, ${ }^{3}$ the most common adverse reaction associated with viscosupplementation is the occurrence of a local reaction at the injection site. This reaction is typically mild and self-limited, resolving within 1-3 days. Meta-analysis has suggested that higher-molecular weight HA products are associated with a greater frequency of local acute inflammatory flares. ${ }^{20}$ Among the 89 trials included in a recent meta-analysis to assess the benefits and risks of viscosupplementation, there were six trials (811 patients total) that evaluated the risk of local flare reaction. Compared with control groups, the risk of local flare reaction with viscosupplementation was not significantly increased (relative risk 1.51; confidence interval [CI] 0.84, 2.72). ${ }^{21}$

Another type of inflammatory response, a severe acute reaction called "pseudosepsis," has been described, particularly after repeat injection with certain HA products. Goldberg and Coutts $^{22}$ define pseudosepsis by five classic clinical criteria: (1) marked inflammation of the joint, typically with significant effusion and pain, normally occurring within 24 to 72 hours after injection; (2) occurring more often after exposure to more than one injection; (3) sepsis or pseudogout are ruled out by the absence of infectious agents and calcium pyrophosphate crystals in the synovial fluid; (4) synovial fluid may include high numbers of mononuclear cells (largely macrophages, with occasional neutrophils, and an increased percentage 
of eosinophils) infiltrating from the surrounding membrane; and (5) pseudosepsis is generally not self-limited and requires clinical intervention, usually in the form of NSAIDs, or arthrocentesis and intraarticular steroid injection. While the exact pathogenesis of pseudosepsis is not known, there is evidence to suggest an immune-mediated etiology. ${ }^{22}$ Early reports attributed the occurrence of pseudosepsis specifically to cross-linked HA derivatives. However, a recent case report described a pseudosepsis-like reaction after a single injection with Curavisc ${ }^{\circledR}$ (Curasan AG, Kleinostheim, Germany), a naturally-derived HA compound produced through biologic fermentation. ${ }^{23}$

\section{Efficacy}

The ideal candidate for viscosupplementation has not yet been clearly defined. ${ }^{5}$ The majority of clinical studies published have predominantly included patients over the age of 60 years, with moderate to severe arthritis. However, a recent meta-analysis found patients over the age of 65 with more advanced radiographic disease at baseline, especially the complete loss of joint space, to be less likely to respond to viscosupplementation than the younger patient population with more moderate disease. ${ }^{24}$

While the efficacy of viscosupplementation has been well supported in the literature, the reader must view the magnitude of effectiveness with some degree of skepticism because of limitations presented by low trial quality and publication bias. ${ }^{19,25}$ For example, the authors of a 2009
Cochrane review ${ }^{3}$ reported that viscosupplementation is an effective treatment for knee OA, with beneficial effects on pain, function, and patient global assessment, especially at the 5- to 13-week period. However, the report revealed that the magnitude of clinical effect is product-dependent. Nonetheless, few randomized, head-to-head trials exist, making direct comparison of different HA products difficult. The reader should also note that recommendations from the Agency for Healthcare Research and Quality ${ }^{25}$ and the American Academy of Orthopaedic Surgeons ${ }^{26}$ related to the use of intraarticular HA for knee OA are inconclusive, largely because poor study design and publication bias have introduced uncertainty of the clinical results.

A recent meta-analysis of randomized trials provides an updated pooled analysis of the effectiveness of intraarticular HA, compared with placebo saline injections, to relieve pain. ${ }^{27}$ There were 7545 patients in the 54 eligible trials included in the meta-analysis, and $16(30 \%)$ of these trials were deemed "high quality" (at least 100 randomized participants and reporting of intention-to-treat analysis, adequate blinding, and allocation concealment). Sensitivity analyses for molecular weight and origin of the HA agent were included in the meta-regression in an attempt to account for their potential influence on effectiveness. The effect size (ES) for relief of joint pain favored HA over placebo by 4 weeks (ES $0.31 ; 95 \%$ CI: $0.17,0.45$ ), peaked at 8 weeks (ES 0.46; CI: 0.28, 0.65), and declined over the remaining

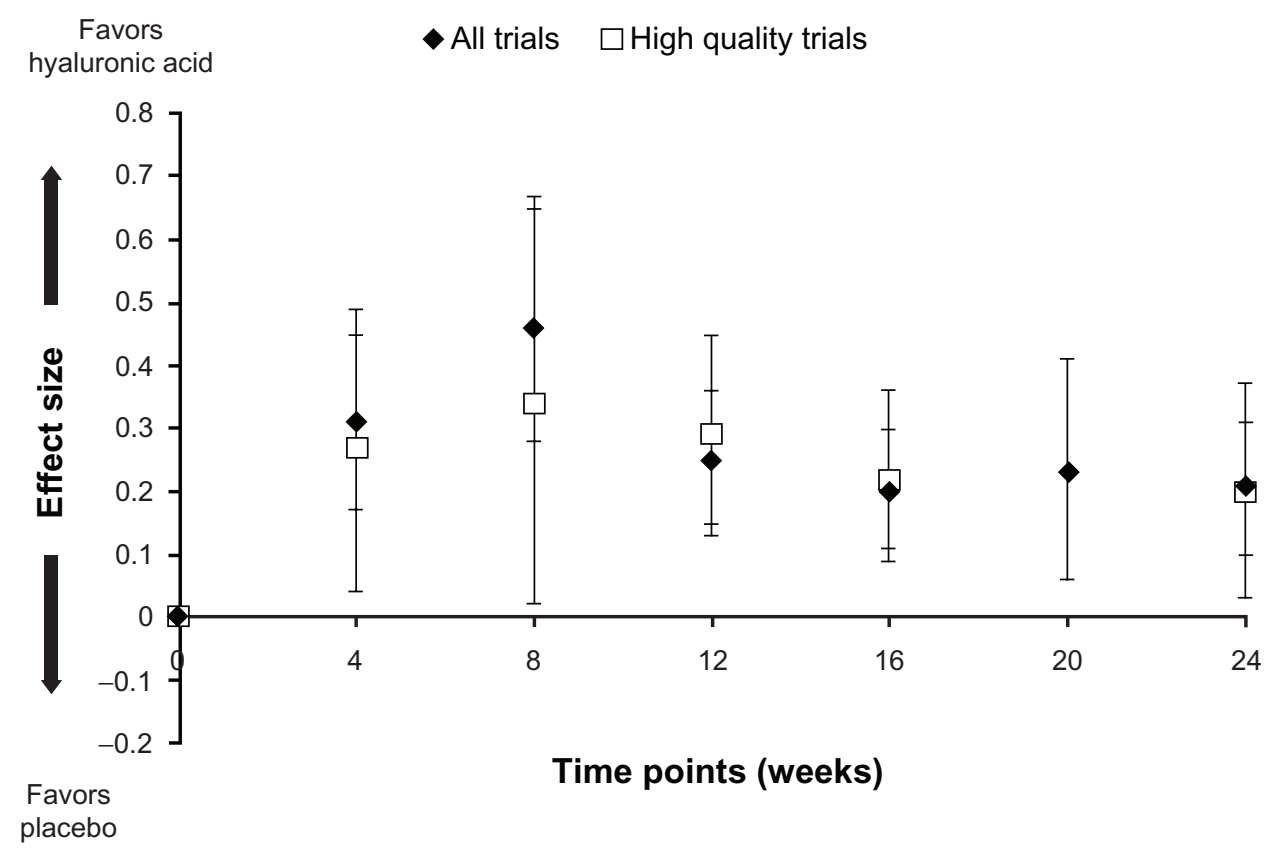

Figure I Effect size (with 95\% confidence intervals) of intraarticular hyaluronic acid for relief of knee pain compared with intraarticular saline (placebo) injection. Note: Reprinted from Osteoarthritis Cartilage, 19(6), Bannuru RR, Natov NS, Dasi UR, Schmid CH, McAlindon TE, Therapeutic trajectory following intra-articular hyaluronic acid injection in knee osteoarthritis - meta-analysis, $61 \mathrm{I}-619$, copyright $201 \mathrm{I}$, with permission from Elsevier. ${ }^{27}$ 
time (Figure 1). At 24 weeks, there was still a statistically significant residual effect (ES 0.21; CI: 0.10, 0.31) of HA compared with placebo. A similar trajectory of pain relief over time was maintained when analyzing only the highquality trials. While its ES is relatively modest (particularly after 24 weeks), HA compares very favorably against other nonoperative treatments for knee $\mathrm{OA}^{27}$ and exceeds an established clinical threshold for relief of chronic knee pain. ${ }^{28}$ Separate analyses have shown no difference in pain relief between intraarticular HA and intraarticular corticosteroids at 4 weeks, but greater effectiveness of HA from 5 to 13 weeks after injection. ${ }^{3,29}$ In another recent meta-analysis of 89 trials, Rutjes et $\mathrm{al}^{21}$ noted a modest ES for knee pain (ES -0.37 ; CI: $-0.46,-0.28)$, which met the pre-specified threshold for minimally clinically important difference.

\section{Patient acceptability}

There is a relative paucity of data regarding patient preferences and the acceptability of a three-injection course of intraarticular HA. Many of the conclusions regarding patient acceptability can be derived from the aforementioned clinical effectiveness in pain relief. With regards to the patient experience of undergoing HA injection, patient satisfaction was assessed at 12 weeks in a study of two HA preparations. The overall satisfaction rate was high, with over $80 \%$ of all patients being either "satisfied" or "very satisfied." ${ }^{30}$ More recently, Briggs et $\mathrm{al}^{31}$ reported on pretreatment patient expectations and posttreatment satisfaction. Relief of pain and improved ability to walk were considered "very important" by $69 \%$ and $78 \%$ of patients, respectively. Nearly all (95\%) patients considered avoidance of future degeneration very important. Median patient satisfaction scores were 8/10 at 3 and 6 weeks and 7/10 at 12 weeks and 6 months.

In our experience, patients exhibit both anxiety and excitement about using a relatively new treatment modality. The physician should have a very careful discussion with the patient to aid in the shared decision-making process, reviewing the sequence of injections, potential for adverse events (namely, local soft tissue reaction, pain flares, and pseudosepsis), and the expected results from both the literature and the physician's personal experience. Although the use of intraarticular HA for knee OA has been demonstrated to be cost effective, ${ }^{32}$ the increased cost of HA (compared with other pharmaceutical treatments, such as acetaminophen and both selective and nonselective Cox-2 inhibitor antiinflammatories) needs to be considered and discussed with the patient. Depending on the structure of the health care system, at least part of this incremental cost may be conferred to the patient. This should be clarified with the patient and/ or payer prior to initiation of the injection.

\section{Future directions}

The encouraging results seen with viscosupplementation in knee OA have led to a number of recent studies assessing the role of intraarticular HA injections for other arthritic joints. While viscosupplementation is currently only approved by the United States Food and Drug Administration (FDA) for the treatment of painful knee OA, there is a growing body of Level 1 evidence to support its role for other joints. Recently published randomized controlled trials have demonstrated efficacy of viscosupplementation in the shoulder, ${ }^{33}$ ankle, ${ }^{34,35}$ and hip. ${ }^{35}$ Evidence for viscosupplementation for trapeziometacarpal (basal joint) arthritis is conflicting. A pilot study of 32 patients demonstrated improvement in pain and function at 26 weeks, ${ }^{36}$ yet a 60 -patient, randomized, controlled trial demonstrated no statistically significant difference from results obtained with corticosteroid or placebo. ${ }^{37}$ The trials involving the hip and ankle joints were relatively small, however, research is ongoing and larger trials can be expected in the years to come. ${ }^{38}$ Both physicians and researchers should be wary of the publication bias and occasional poor trial quality in the previously published literature when planning future studies, as both may lead to an overestimation of the effectiveness of intraarticular HA in providing pain relief. ${ }^{19,25}$

The chondroprotective and inductive properties that have been attributed to HA injections remain an area of active research. The role of HA supplementation in restoring a favorable intraarticular milieu after arthroscopic procedures has been an area of particular interest. Hempfling ${ }^{39}$ noted improved outcomes in pain relief and restoration of function among patients who received HA injection following knee arthroscopy. A number of recent clinical and animal studies have focused on the potential role for viscosupplementation after a number of arthroscopic procedures, including meniscal repair, ${ }^{40,41}$ ACL reconstruction, ${ }^{42}$ and treatment of osteochondral defects. ${ }^{43,44}$

With regards to the treatment of osteoarthritis, the potential for disease-modifying therapy has been suggested by several in vitro and in vivo studies. ${ }^{9}$ More than a simple mechanical lubricant and shock-absorber, intraarticular HA injection has been shown to alter the biology of the joint at the molecular level, and a number of animal studies have demonstrated evidence of reduced cartilage degeneration and promotion of tissue repair with HA injection. ${ }^{9,45-56}$ Furthermore, recent clinical studies suggest that HA injection may be associated with slowed progression of disease, ${ }^{57}$ 
reparative structural changes,${ }^{58}$ and induction of endogenous HA production. ${ }^{59}$

\section{Conclusion}

As the prevalence of osteoarthritis is projected to increase dramatically in the coming years, the importance of safe, effective, and acceptable therapies is increasingly apparent. Viscosupplementation with intraarticular HA has demonstrated a favorable safety profile and clinical effectiveness in relieving pain for a temporary period, with high levels of overall patient satisfaction. Physicians must counsel patients that while intraarticular HA is effective, its benefits are temporary and that patients may eventually require further treatment, including surgical management.

\section{Disclosure}

Dr Gonzalez Della Valle's efforts on this study were generously funded through an unrestricted philanthropic donation from Mr Glenn Bergenfield and The Simon Foundation. None of the other authors have any financial agreements or conflicts of interest regarding the products discussed in this article.

\section{References}

1. Stewart WF, Ricci JA, Chee E, Morganstein D, Lipton R. Lost productive time and cost due to common pain conditions in the US workforce. JAMA. 2003;290(18):2443-2454.

2. Hootman JM, Helmick CG. Projections of US prevalence of arthritis and associated activity limitations. Arthritis Rheum. 2006;54(1): 226-229.

3. Bellamy N, Campbell J, Robinson V, Gee T, Bourne R, Wells G. Viscosupplementation for the treatment of osteoarthritis of the knee. Cochrane Database Syst Rev. 2006;2:CD005321.

4. Goldberg VM, Goldberg L. Intra-articular hyaluronans: the treatment of knee pain in osteoarthritis. J Pain Res. 2010;3:51-56.

5. Strauss EJ, Hart JA, Miller MD, Altman RD, Rosen JE. Hyaluronic acid viscosupplementation and osteoarthritis: current uses and future directions. Am J Sports Med. 2009;37(8):1636-1644.

6. Brockmeier SF, Shaffer BS. Viscosupplementation therapy for osteoarthritis. Sports Med Arthrosc. 2006;14(3):155-162.

7. Altman RD, Rosen JE, Bloch DA, Hatoum HT, Korner P. A doubleblind, randomized, saline-controlled study of the efficacy and safety of EUFLEXXA for treatment of painful osteoarthritis of the knee, with an open-label safety extension (the FLEXX trial). Semin Arthritis Rheum. 2009;39(1):1-9.

8. Watterson JR, Esdaile JM. Viscosupplementation: therapeutic mechanisms and clinical potential in osteoarthritis of the knee. J Am Acad Orthop Surg. 2000;8(5):277-284.

9. Goldberg VM, Buckwalter JA. Hyaluronans in the treatment of osteoarthritis of the knee: evidence for disease-modifying activity. Osteoarthritis Cartilage. 2005;13(3):216-224.

10. Moreland LW. Intra-articular hyaluronan (hyaluronic acid) and hylans for the treatment of osteoarthritis: mechanisms of action. Arthritis Res Ther. 2003;5(2):54-67.

11. Jean YH, Wen ZH, Chang YC, et al. Increased concentrations of neuroexcitatory amino acids in rat anterior cruciate ligament-transected knee joint dialysates: a microdialysis study. J Orthop Res. 2005;23(3): 569-575.
12. Jean YH, Wen $\mathrm{ZH}$, Chang YC, et al. Hyaluronic acid attenuates osteoarthritis development in the anterior cruciate ligament-transected knee: Association with excitatory amino acid release in the joint dialysate. J Orthop Res. 2006;24(5):1052-1061.

13. Tsai W, Liu C, Cherng C, et al. Early intraarticular injection of hyaluronic acid attenuates osteoarthritis progression in anterior cruciate ligament-transected rats. Connect Tissue Res. Epub September 28, 2012.

14. Kawasaki K, Ochi M, Uchio Y, Adachi N, Matsusaki M. Hyaluronic acid enhances proliferation and chondroitin sulfate synthesis in cultured chondrocytes embedded in collagen gels. J Cell Physiol. 1999;179(2):142-148.

15. Kujawa MJ, Caplan AI. Hyaluronic acid bonded to cell-culture surfaces stimulates chondrogenesis in stage 24 limb mesenchyme cell cultures. Dev Biol. 1986;114(2):504-518.

16. Aviad AD, Houpt JB. The molecular weight of therapeutic hyaluronan (sodium hyaluronate): how significant is it? J Rheumatol. 1994;21(2): 297-301.

17. Lo GH, LaValley M, McAlindon T, Felson DT. Intra-articular hyaluronic acid in treatment of knee osteoarthritis: a meta-analysis. JAMA. 2003;290(23):3115-3121.

18. Maneiro E, de Andres MC, Fernández-Sueiro JL, Galdo F, Blanco FJ. The biological action of hyaluronan on human osteoartritic articular chondrocytes: the importance of molecular weight. Clin Exp Rheumatol. 2004;22(3):307-312.

19. Zhang W, Nuki G, Moskowitz RW, et al. OARSI recommendations for the management of hip and knee osteoarthritis: part III: Changes in evidence following systematic cumulative update of research published through Jan 2009. Osteoarthritis Cartilage. 2010;18(4):476-499.

20. Reichenbach S, Blank S, Rutjes AW, et al. Hylan versus hyaluronic acid for osteoarthritis of the knee: a systematic review and meta-analysis. Arthritis Rheum. 2007;57(8):1410-1418.

21. Rutjes AW, Jüni P, da Costa BR, Trelle S, Nüesch E, Reichenbach S. Viscosupplementation for osteoarthritis of the knee: a systematic review and meta-analysis. Ann Intern Med. 2012;157(3):180-191.

22. Goldberg VM, Coutts RD. Pseudoseptic reactions to hylan viscosupplementation: diagnosis and treatment. Clin Orthop Relat Res. 2004;419:130-137.

23. Tahiri L, Benbouazza K, Amine B, Hajjaj-Hassouni N. Acute pseudoseptic arthritis after viscosupplementation of the knee: a case report. Clin Rheumatol. 2007;26(11):1977-1979.

24. Wang CT, Lin J, Chang CJ, Lin YT, Hou SM. Therapeutic effects of hyaluronic acid on osteoarthritis of the knee. A meta-analysis of randomized controlled trials. J Bone Joint Surg Am. 2004;86-A(3):538-545.

25. Samson DJ, Grant MD, Ratko TA, Bonnell CJ, Ziegler KM, Aronson N. Treatment of Primary and Secondary Osteoarthritis of the Knee. Rockville: Agency for Healthcare Research and Quality; 2007. [cited May 7, 2012]. Available from: http://archive.ahrq.gov/downloads/ pub/evidence/pdf/oaknee/oaknee.pdf. Accessed November 9, 2012.

26. Richmond J, Hunter D, Irrgang J, et al; American Academy of Orthopaedic Surgeons. Treatment of osteoarthritis of the knee (nonarthroplasty). J Am Acad Orthop Surg. 2009;17(9):591-600.

27. Bannuru RR, Natov NS, Dasi UR, Schmid CH, McAlindon TE. Therapeutic trajectory following intra-articular hyaluronic acid injection in knee osteoarthritis - meta-analysis. Osteoarthritis Cartilage. 2011; 19(6):611-619.

28. Dworkin RH, Turk DC, Wyrwich KW, et al. Interpreting the clinical importance of treatment outcomes in chronic pain clinical trials: IMMPACT recommendations. J Pain. 2008;9(2):105-121.

29. Bannuru RR, Natov NS, Obadan IE, Price LL, Schmid CH, McAlindon TE. Therapeutic trajectory of hyaluronic acid versus corticosteroids in the treatment of knee osteoarthritis: a systematic review and meta-analysis. Arthritis Rheum. 2009;61(12):1704-1711.

30. Kirchner M, Marshall D. A double-blind randomized controlled trial comparing alternate forms of high molecular weight hyaluronan for the treatment of osteoarthritis of the knee. Osteoarthritis Cartilage. 2006;14(2):154-162. 
31. Briggs KK, Matheny LM, Steadman JR. Can hylan g-f 20 with corticosteroid meet the expectations of osteoarthritis patients? Am J Orthop (Belle Mead NJ). 2012;41(7):311-315.

32. Torrance GW, Raynauld JP, Walker V, et al; Canadian Knee OA Study Group. A prospective, randomized, pragmatic, health outcomes trial evaluating the incorporation of hylan G-F 20 into the treatment paradigm for patients with knee osteoarthritis (Part 2 of 2): economic results. Osteoarthritis Cartilage. 2002;10(7):518-527.

33. Blaine T, Moskowitz R, Udell J, et al. Treatment of persistent shoulder pain with sodium hyaluronate: a randomized, controlled trial. A multicenter study. J Bone Joint Surg Am. 2008;90(5):970-979.

34. Cohen MM, Altman RD, Hollstrom R, Hollstrom C, Sun C, Gipson B. Safety and efficacy of intra-articular sodium hyaluronate (Hyalgan) in a randomized, double-blind study for osteoarthritis of the ankle. Foot Ankle Int. 2008;29(7):657-663.

35. Migliore A, Massafra U, Bizzi E, et al. Comparative, double-blind, controlled study of intra-articular hyaluronic acid (Hyalubrix) injections versus local anesthetic in osteoarthritis of the hip. Arthritis Res Ther. 2009;11(6):R183.

36. Mandl LA, Hotchkiss RN, Adler RS, et al. Injectable hyaluronan for the treatment of carpometacarpal osteoarthritis: open label pilot trial. Curr Med Res Opin. 2009;25(9):2103-2108.

37. Heyworth BE, Lee JH, Kim PD, Lipton CB, Strauch RJ, Rosenwasser MP. Hylan versus corticosteroid versus placebo for treatment of basal joint arthritis: a prospective, randomized, double-blinded clinical trial. $J$ Hand Surg Am. 2008;33(1):40-48.

38. Colen S, van den Bekerom MP, Bellemans J, Mulier M. Comparison of intra-articular injections of hyaluronic acid and corticosteroid in the treatment of osteoarthritis of the hip in comparison with intra-articular injections of bupivacaine. Design of a prospective, randomized, controlled study with blinding of the patients and outcome assessors. BMC Musculoskelet Disord. 2010;11:264.

39. Hempfling $\mathrm{H}$. Intra-articular hyaluronic acid after knee arthroscopy: a two-year study. Knee Surg Sports Traumatol Arthrosc. 2007;15(5): $537-546$.

40. Ishima M, Wada Y, Sonoda M, Harada Y, Katsumi A, Moriya H. Effects of hyaluronan on the healing of rabbit meniscus injured in the peripheral region. J Orthop Sci. 2000;5(6):579-584.

41. Suzuki Y, Takeuchi N, Sagehashi Y, Yamaguchi T, Itoh H, Iwata H. Effects of hyaluronic acid on meniscal injury in rabbits. Arch Orthop Trauma Surg. 1998;117(6-7):303-306.

42. Huang MH, Yang RC, Chou PH. Preliminary effects of hyaluronic acid on early rehabilitation of patients with isolated anterior cruciate ligament reconstruction. Clin J Sport Med. 2007;17(4):242-250.

43. Miot $\mathrm{S}, \mathrm{Brehm} \mathrm{W}$, Dickinson $\mathrm{S}$, et al. Influence of in vitro maturation of engineered cartilage on the outcome of osteochondral repair in a goat model. Eur Cell Mater. 2012;23:222-236.

44. Suwannaloet W, Laupattarakasem W, Sukon P, Ong-Chai S, Laupattarakasem P. Combined effect of subchondral drilling and hyaluronic acid with/without diacerein in full-thickness articular cartilage lesion in rabbits. Scientific World Journal. 2012;2012:310745.
45. Takahashi K, Hashimoto S, Kubo T, Hirasawa Y, Lotz M, Amiel D. Effect of hyaluronan on chondrocyte apoptosis and nitric oxide production in experimentally induced osteoarthritis. J Rheumatol. 2000;27(7): $1713-1720$.

46. Yoshioka M, Shimizu C, Harwood FL, Coutts RD, Amiel D. The effects of hyaluronan during the development of osteoarthritis. Osteoarthritis Cartilage. 1997;5(4):251-260.

47. Shimizu C, Yoshioka M, Coutts RD, et al. Long-term effects of hyaluronan on experimental osteoarthritis in the rabbit knee. Osteoarthritis Cartilage. 1998;6(1):1-9.

48. Kikuchi T, Yamada H, Shimmei M. Effect of high molecular weight hyaluronan on cartilage degeneration in a rabbit model of osteoarthritis. Osteoarthritis Cartilage. 1996;4(2):99-110.

49. Sonoda M, Harwood FL, Amiel ME, et al. The effects of hyaluronan on tissue healing after meniscus injury and repair in a rabbit model. Am J Sports Med. 2000;28(1):90-97.

50. Sonoda M, Harwood FL, Wada Y, Moriya H, Amiel D. The effects of hyaluronan on the meniscus and on the articular cartilage after partial meniscectomy. Am J Sports Med. 1997;25(6):755-762.

51. Armstrong S, Read R, Ghosh P. The effects of intraarticular hyaluronan on cartilage and subchondral bone changes in an ovine model of early osteoarthritis. J Rheumatol. 1994;21(4):680-688.

52. Wiig ME, Amiel D, VandeBerg J, Kitabayashi L, Harwood FL, Arfors KE. The early effect of high molecular weight hyaluronan (hyaluronic acid) on anterior cruciate ligament healing: an experimental study in rabbits. J Orthop Res. 1990;8(3):425-434.

53. Amiel D, Toyoguchi T, Kobayashi K, Bowden K, Amiel ME, Healey RM. Long-term effect of sodium hyaluronate (Hyalgan) on osteoarthritis progression in a rabbit model. Osteoarthritis Cartilage. 2003;11(9):636-643.

54. Marshall KW, Manolopoulos V, Mancer K, Staples J, Damyanovich A. Amelioration of disease severity by intraarticular hylan therapy in bilateral canine osteoarthritis. J Orthop Res. 2000;18(3):416-425.

55. Schiavinato A, Lini E, Guidolin D, et al. Intraarticular sodium hyaluronate injections in the Pond-Nuki experimental model of osteoarthritis in dogs. II. Morphological findings. Clin Orthop Relat Res. 1989;241:286-299.

56. Wenz W, Breusch SJ, Graf J, Stratmann U. Ultrastructural findings after intraarticular application of hyaluronan in a canine model of arthropathy. J Orthop Res. 2000;18(4):604-612.

57. Listrat V, Ayral X, Patarnello F, et al. Arthroscopic evaluation of potential structure modifying activity of hyaluronan (Hyalgan) in osteoarthritis of the knee. Osteoarthritis Cartilage. 1997;5(3):153-160.

58. Pasquali Ronchetti I, Guerra D, Taparelli F, et al. Morphological analysis of knee synovial membrane biopsies from a randomized controlled clinical study comparing the effects of sodium hyaluronate (Hyalgan) and methylprednisolone acetate (Depomedrol) in osteoarthritis. Rheumatology (Oxford). 2001;40(2):158-169.

59. Bagga H, Burkhardt D, Sambrook P, March L. Longterm effects of intraarticular hyaluronan on synovial fluid in osteoarthritis of the knee. J Rheumatol. 2006;33(5):946-950.
Patient Preference and Adherence

\section{Publish your work in this journal}

Patient Preference and Adherence is an international, peer-reviewed, open access journal focusing on the growing importance of patient preference and adherence throughout the therapeutic continuum. Patient satisfaction, acceptability, quality of life, compliance, persistence and their role in developing new therapeutic modalities and compounds to

\section{Dovepress}

optimize clinical outcomes for existing disease states are major areas of interest. This journal has been accepted for indexing on PubMed Central. The manuscript management system is completely online and includes a very quick and fair peer-review system. Visit http://www.dovepress.com/ testimonials.php to read real quotes from published authors. 